\title{
Investigación Cualitativa: Una respuesta a las Investigaciones Sociales Educativas
}

\section{Qualitative Research: A Response to Educational Social Research}

\author{
Lucy Stella Salazar-Escorcia \\ lucystella75@gmail.com \\ Institución Técnico Agropecuario La Arena, Sincelejo \\ Colombia \\ https://orcid.org/0000-0003-0300-8131
}

Recibido: 24 de enero de 2020

Aprobado: 26 de febrero de 2020

\begin{abstract}
RESUMEN
Los acercamientos de tipo cualitativo reivindican el abordaje de las realidades subjetiva e intersubjetiva como objetos legítimos de conocimiento científico; el estudio de la vida cotidiana como el escenario básico de construcción, constitución y desarrollo de los distintos planos que configuran e integran las dimensiones específicas del mundo humano y, por último, ponen de relieve el carácter único, multifacético y dinámico de las realidades humanas. Por esta vía emerge, la necesidad de ocuparse de problemas como la libertad, moralidad y la significación de las acciones humanas, dentro de un proceso de construcción socio-cultural e histórica, cuya comprensión es clave para acceder a un conocimiento pertinente y válido de lo humano. La investigación fue sustentada en autores como: Domínguez (2000), Arias (2012) Vera (2015), UPEL (2006) entre otros. La metodología utilizada fue documental con nivel descriptivo. Por último, se evidencia el papel que tiene la investigación en el contexto educativo.
\end{abstract}

Descriptores: Investigación; educación; cualitativa; ciencias, sociales.

\begin{abstract}
The qualitative approaches claim the approach of subjective and intersubjective realities as legitimate objects of scientific knowledge; the study of daily life as the basic stage of construction, constitution and development of the different planes that configure and integrate the specific dimensions of the human world and, finally, highlight the unique, multifaceted and dynamic character of human realities. In this way, the need to deal with problems such as freedom, morality and the significance of human actions emerges, within a process of socio-cultural and historical construction,
\end{abstract}




\section{CIENCIAMATRIA}

Revista Interdisciplinaria de Humanidades, Educación, Ciencia y Tecnología

Año VI. Vol. VI. N¹1. Julio - Diciembre 2020

Hecho el depósito de ley: pp201602FA4721

ISSN-L: 2542-3029; ISSN: 2610-802X

Universidad Nacional Experimental Francisco de Miranda (UNEFM). Santa Ana de Coro. Venezuela

Lucy Stella Salazar-Escorcia

whose understanding is key to accessing relevant and valid knowledge of what human. The research was supported by authors such as: Dominguez (2000), Arias (2012) Vera (2015), UPEL (2006), among others. The methodology used was documentary with a descriptive level. Finally, the role of research in the educational context is evident.

Descriptors: Research, education, qualitative, science, social.

\section{INTRODUCCIÓN}

Para comprender la caracterización metodológica de una opción investigativa, resulta necesario y conveniente indagar por sus bases epistemológicas, de modo que se halle el sentido o la razón de ser de sus procedimientos para producir conocimiento científico. Siguiendo con el argumento anterior, el abordaje de los enfoques de investigación en el terreno de las ciencias sociales busca establecer cuáles son las ópticas que se han desarrollado para concebir y mirar las distintas realidades que componen el orden de lo humano, así como también comprender la lógica de los caminos, que se han construido para producir, intencionada y metódicamente conocimiento sobre ellas. En relación con esto último, Taylor y Bogdan (1992) señalan que lo que define la metodología es simultáneamente tanto la manera cómo enfocamos los problemas, como la forma en que le buscamos las respuestas a los mismos. En un planteamiento relacionado, Guba (1990) logra una interesante síntesis, que permite diferenciar los enfoques o paradigmas de investigación social, a partir de la respuesta a tres preguntas básicas: ¿Cómo se concibe la naturaleza tanto del Conocimiento como de la realidad? ¿Cómo se concibe la naturaleza de las relaciones entre el investigador y el conocimiento que genera? ¿Cuál es el modo en que construye o desarrolla conocimiento el investigador?

De allí entonces que el artículo en esta oportunidad está estructurado de la siguiente manera: como primera parte una introducción a la temática, de igual manera se rige por una fundamentación teórica donde se describen aspectos relacionados con los aspectos más importantes y de interés sobre la investigación cualitativa, describiéndose el método de investigación para llegar entonces a concluir con unas consideraciones finales. 


\section{CIENCIAMATRIA}

Revista Interdisciplinaria de Humanidades, Educación, Ciencia y Tecnología

Año VI. Vol. VI. N¹1. Julio - Diciembre 2020

Hecho el depósito de ley: pp201602FA4721

ISSN-L: 2542-3029; ISSN: 2610-802X

Universidad Nacional Experimental Francisco de Miranda (UNEFM). Santa Ana de Coro. Venezuela

Lucy Stella Salazar-Escorcia

\section{DESARROLLO TEÓRICO}

\section{Definiciones de Investigación Cualitativa por autores}

El término "cualitativo", ordinariamente, se usa bajo dos acepciones. Una, como cualidad: "fulano tiene una gran cualidad: es sincero". $Y$ otra, más integral y comprehensiva, como cuando nos referimos al "control de calidad", donde la calidad representa la naturaleza y esencia completa y total de un producto. Cualidad y Calidad vienen del mismo término latino qualitas, y éste deriva de qualis (cuál, qué). De modo que a la pregunta por la naturaleza o esencia de un ser: ¿qué es?, ¿cómo es?, se da la respuesta señalando o describiendo su conjunto de cualidades o la calidad del mismo.

Domínguez (2000) señala que los métodos cualitativos parten del supuesto básico de que el mundo social está construido de significados y símbolos. De ahí que la intersubjetividad sea una pieza clave de la investigación cualitativa y punto de partida para captar reflexivamente los significados sociales. La realidad social así vista está hecha de significados compartidos de manera intersubjetiva. El objetivo y lo objetivo es el sentido intersubjetivo que se atribuye a una acción. La investigación cualitativa puede ser vista como el intento de obtener una comprensión profunda de los significados y definiciones de la situación tal como nos la presentan las personas, más que la producción de una medida cuantitativa de sus características o conducta.

Por su parte Vera (2015) expresa que la investigación cualitativa es aquella donde se estudia la calidad de las actividades, relaciones, asuntos, medios, materiales o instrumentos en una determinada situación o problema. La misma procura por lograr una descripción holística, esto es, que intenta analizar exhaustivamente, con sumo detalle, un asunto $o$ actividad en particular.

De igual modo Hernández y otros (2010) refieren que parte de un esquema inductivo expansivo se utiliza para descubrir y refinar preguntas de investigación, se basa en descripciones y observaciones. Parte de la premisa de toda cultura o sistema social para entender cosas y eventos; su propósito es reconstruir la realidad, tal como la observan los investigadores. Se llama holístico, porque considera el todo, sin reducirlo 


\section{CIENCIAMATRIA}

Revista Interdisciplinaria de Humanidades, Educación, Ciencia y Tecnología

Año VI. Vol. VI. N¹1. Julio - Diciembre 2020

Hecho el depósito de ley: pp201602FA4721

ISSN-L: 2542-3029; ISSN: 2610-802X

Universidad Nacional Experimental Francisco de Miranda (UNEFM). Santa Ana de Coro. Venezuela

Lucy Stella Salazar-Escorcia

a sus partes. Es individual. Su método de análisis es el interpretativo, contextual y etnográfico.

Asimismo Báez (2009) la define como "una categoría de diseños de investigación que extraen descripciones a partir de observaciones que adoptan la forma de entrevistas, narraciones, notas de campo, grabaciones, transcripciones de audio y vídeocassettes, registros escritos de todo tipo, fotografías o películas y artefactos

Por tanto puede decirse que la investigación cualitativa centra su atención en buscar en los fenómenos todas esas cualidades, características y aspectos importantes que le permitan reconstruir la realidad observada y detectada por el investigador a partir de las diferentes técnicas de recolección de datos que la misma investigación presenta; este proceso debería de ser lo más objetivo posible de manera de que la información que emerge sea la más fidedigna.

\section{Métodos de la Investigación Cualitativa.}

En palabras de Báez (2010) en este tipo de investigación se utilizan los siguientes métodos: Fenomenología: busca conocer los significados que los individuos dan a su experiencia y lo importante es aprender el proceso de interpretación por el que la gente define su mundo y actúa en consecuencia. Etnografía: la entendemos como el método de investigación por el que se aprende el modo de vida de una unidad social concreta. Teoría fundamentada: trata de descubrir teorías, conceptos, hipótesis y proposiciones partiendo directamente de los datos, y no de supuestos a priori, de otras investigaciones o de marcos teóricos existentes. Etnometodología: intenta estudiar los fenómenos sociales incorporados a nuestros discursos y nuestras acciones a través del análisis de las actividades humanas. Investigación-acción: es una forma de búsqueda autorreflexiva para perfeccionar la lógica y la equidad de las propias prácticas sociales o educativas que se efectúan estas prácticas, comprensión de estas prácticas y las situaciones en las que se efectúan estas prácticas. Método biográfico: pretende mostrar el testimonio subjetivo de una persona en la que se recojan tanto los acontecimientos como las valoraciones que dicha persona hace de su propia existencia. 


\section{CIENCIAMATRIA}

Revista Interdisciplinaria de Humanidades, Educación, Ciencia y Tecnología

Año VI. Vol. VI. N¹1. Julio - Diciembre 2020

Hecho el depósito de ley: pp201602FA4721

ISSN-L: 2542-3029; ISSN: 2610-802X

Universidad Nacional Experimental Francisco de Miranda (UNEFM). Santa Ana de Coro. Venezuela

Lucy Stella Salazar-Escorcia

\section{Fases y técnicas de la Investigación Cualitativa}

Respecto al proceso y las fases de la investigación cualitativa, se diferencian cinco fases de trabajo: 1. Definición del problema. 2. Diseño de trabajo. 3. Recogida de datos. 4. Análisis de datos. 5. Informe y validación de la información.

Además, dentro de la investigación cualitativa podemos encontrar diversas técnicas como: 1. La observación. 2. La observación participante. 3. La entrevista. 4. La entrevista grupal. 5. El cuestionario. 6. El grupo de discusión El informe de resultados es el cierre final de la investigación emprendida. Es la fase de la investigación que pone en relación la demanda o problema que dio pie al estudio con los resultados obtenidos. Su carácter paradójico, de final y principio a la vez, hace que en ocasiones se considere un producto a consumir y en otras situaciones un proceso a desarrollar. Lo que permite el informe cualitativo es facilitar y ampliar la investigación a un ámbito metodológico distinto, incluso como forma de mejorar la aplicación de las mismas técnicas de investigación.

En cuanto a la elaboración del informe, es importante incluir los contextos o circunstancias concretas en las que se desarrolló la investigación que se documenta. Los tres contextos fundamentales son; el contexto de la demanda, el contexto del trabajo decampo y el contexto de la población. Hay cuatro tipos de informes y sus correspondientes receptores: el informe científico, el informe técnico, el informe ejecutivo y el informe divulgativo. Cada tipo de informe pone el acento en una finalidad o propósito acorde con los requerimientos de la demanda que encarga el estudio.

El informe cualitativo no puede incluir toda la información que sería de desear para documentar sus hallazgos y hacerlos más verosímiles, debe mantener cierta unidad y coherencia interna en la exposición lógica de los resultados. El informe debe atender a dos cuestiones básicas para su correcta elaboración. En primer lugar, tener en cuenta qué uso se le va a dar desde el punto de vista de demanda y qué circunstancias concretas dieron un sentido práctico y realista al diseño de la investigación.

En segundo lugar, el informe debe tener en cuenta como presentar dicha investigación de manera que resulte lo más clara y atractiva para el potencial lector. A la hora de redactar el informe es necesario tener en cuenta varios requisitos: No es apropiado 


\section{CIENCIAMATRIA}

Revista Interdisciplinaria de Humanidades, Educación, Ciencia y Tecnología

Año VI. Vol. VI. N¹1. Julio - Diciembre 2020

Hecho el depósito de ley: pp201602FA4721

ISSN-L: 2542-3029; ISSN: 2610-802X

Universidad Nacional Experimental Francisco de Miranda (UNEFM). Santa Ana de Coro. Venezuela

Lucy Stella Salazar-Escorcia

utilizar una terminología sofisticada. Es aconsejable utilizar tiempos verbales en presente para redactar las ideas del informe. Es oportuno utilizar expresiones de advertencia en términos de probabilidad Deberá hacerse explícito lo que se va a exponer cómo se va a exponer y con qué finalidad o propósito se expone. El informe debe seguir un hilo conductor que ordene y contribuya a dar una lógica y coherencia interna al conjunto de la exposición. Las representaciones más utilizadas en la investigación social cualitativa son: los mapas, las tablas y los esquemas.

Una vez que contamos con toda la información reunida y ha terminado el trabajo de campo estamos en condiciones de realizar el análisis de los datos. El sentido del análisis de datos en la investigación cualitativa consiste en reducir, categorizar, clarificar, sintetizar y comparar la información con el fin de obtener una visión lo más completa posible de la realidad objeto de estudio. Esta etapa no es algo que se lleva a cabo al final de la investigación, sino que es una tarea que se realiza a lo largo de todo el proceso de la misma

\section{METODOLOGÍA}

En relación a la metodología del estudio, ésta tiene sus orígenes en el método científico, basado en la unificación de un conjunto de conocimientos epistémicos conectados entre sí, la descripción analítica de sucesos y las teorías de autores, el enfoque de la misma es cualitativo, en este sentido se acerca al entorno de la realidad, dejando ver la razón de ser y el comportamiento de la situación de estudio.

De acuerdo a lo anterior, Báez y Pérez (2009), plantean que un enfoque epistémico representa un modelo a seguir, aludiendo que la noción de este paradigma en las ciencias sociales, va más asociada a los valores que condicionan la percepción del entorno social, por lo que este constituye una serie de creencias además de actitudes, ya que se trata de la visión que un equipo de investigadores que comparten puntos de vistas.

Es por ello que de acuerdo a la naturaleza de las fuentes utilizadas, la presente investigación se realizó mediante un diseño documental, Según Arias (2012 p 34) "la investigación documental es un proceso basado en la búsqueda, recuperación, 


\section{CIENCIAMATRIA}

Revista Interdisciplinaria de Humanidades, Educación, Ciencia y Tecnología

Año VI. Vol. VI. N¹1. Julio - Diciembre 2020

Hecho el depósito de ley: pp201602FA4721

ISSN-L: 2542-3029; ISSN: 2610-802X

Universidad Nacional Experimental Francisco de Miranda (UNEFM). Santa Ana de Coro. Venezuela

Lucy Stella Salazar-Escorcia

análisis, critica e interpretación de datos secundarios, es decir, los obtenidos y registrados por otros investigadores en fuentes documentales impresas, audiovisuales o electrónicas".

De igual modo el Manual de la UPEL (2006) define la investigación documental y bibliográfica como aquellas revisiones críticas del estado del conocimiento: integración, organización y evaluación de la información teórica y empírica existente sobre un problema, focalizando ya sea en el progreso de la investigación actual y posibles vías para su solución, en el análisis de la consistencia interna y externa de las teorías y conceptualizaciones para señalar sus fallas o demostrar su superioridad de unas sobre otras, o en ambos aspectos.

Es asi como de igual modo se hace importante destacar lo planteado por Azuero (2018) cuando indica que la formulación del marco metodológico en una investigación, es permitir, descubrir los supuestos del estudio para reconstruir datos, a partir de conceptos teóricos habitualmente operacionalizados. Significa detallar cada aspecto seleccionado para desarrollar dentro del proyecto de investigación que deben ser justificado por el investigador.

Por lo descrito anteriormente puede concluirse que el diseño de la investigación viene dado por una estructura teórico-conceptual, que conllevo a la revisión exhaustiva de las diferentes fuentes de información, tales como: libros, documentos de diversos organismos nacionales e internacionales, informes, trabajos de grado, revistas científicas, leyes, normas y otra bibliografía relacionada con el tema de estudio.

\section{CONSIDERACIONES FINALES}

Para finalizar es necesario sintetizar con unos elementos $u$ aspectos puntuales que este tipo de investigación nos presenta y que en el ámbito educativo nos puede a ayudar a solventar situaciones del día a día entre los cuales se pueden mencionar:

La característica fundamental de la investigación cualitativa es ver los acontecimientos, acciones, normas, valores, etc., desde la perspectiva de la gente que está siendo estudiada. Sustentada en las tendencias subjetivistas, las que pretenden una comprensión del fenómeno social, concediendo a lo subjetivo la principal fuente 
de los datos; antes que generar leyes universales, buscan la descripción y comprensión de escenario particulares.

El mundo social depende de los sujetos y son ellos quienes los construyen y lo vivencian; por lo tanto, para conocerlo no es suficiente generar explicaciones objetivas sobre él, no es posible considerar que el mundo social este regido por leyes universales, puesto que las realidades son para los sujetos y grupos una realidad diferente. En este sentido, una realidad social no puede cuadricularse en un plano cartesiano y tabla porcentual, puesto que la sociedad es dinámica de significantes y significados que constituyen dicha realidad.

La estrategia de tomar la perspectiva del sujeto, se expresa generalmente en términos de "ver a través de los ojos de la gente que uno está estudiando", tal perspectiva, nos envuelve a usar la empatía, con quienes están siendo estudiados, penetrando los contextos de significado con los cuales ellos operan, por lo tanto, esto nos llevará unos períodos de tiempo. Todo esto justifica el uso de las técnicas de observación participante combinada con la entrevista en profundidad no estructurada.

Un problema clave para el investigador es definir, a través de qué ojos trataré de observar la realidad. La necesidad de tomar la perspectiva del otro implica tomar en cuenta la existencia de una multiplicidad de puntos de vista, de visiones de la realidad. Entre los metodólogos cualitativos, especialmente los etnógrafos, hay un claro propósito de proveer descripciones detalladas de los contextos sociales estudiados. El énfasis en la descripción se basa en que lo aparentemente minucioso o trivial de la vida diaria, es valioso de observar, puesto que puede ayudarnos a entender lo que está pasando en el contexto particular

Describir es complejo, responder a la pregunta ¿Qué está sucediendo aquí?, no es simple. Lo importante es mapear un contexto, para poder entender la interpretación que hace el sujeto acerca de lo que está sucediendo. Esto posibilita al investigador a producir análisis y expresiones que hagan justicia al medio ambiente en que las observaciones son hechas; con lo cual se destaca en la investigación cualitativa la preferencia por la contextualización inseparable del holismo. 
Esta última implica la preferencia por examinar entidades sociales, escuela, tribus, como globalidades a ser aplicadas y entendidas en su integridad. El énfasis es puesto en la necesidad de interpretar qué está pasando en términos de un entendimiento de la sociedad como un todo y del significado que tiene para los participantes; por lo tanto, sólo podemos entender los acontecimientos si ellos son situados en un contexto social e históricamente amplio.

La investigación cualitativa es más que procesar lo que la vida social es vista como una serie de acontecimientos y se hace más énfasis en los cambios que los procesos implican. Todo esto nos permite considerar que la investigación cualitativa favorece a una estrategia de investigación relativamente abierta y no estructurada, más que una en la cual el investigador decide por adelantado lo que va a investigar y cómo lo va a hacer.

De la misma manera es importante señalar lo expuesto por Azuero (2018) cuando indica que la investigación cualitativa atraviesa diversas disciplinas, participa de una gran variedad de discursos o perspectivas teóricas y engloba numerosos métodos y estrategias de recogida de datos. Esta riqueza denota la complejidad y alcance del enfoque cualitativo en el abordaje de la investigación socioeducativa y requiere que se ensayen clasificaciones o categorías que aporten un orden conceptual en el ámbito investigación y permitan la comunicación en la comunidad investigadora.

Por todo lo expuesto, en la investigación cualitativa se rechaza la formulación de teorías y conceptos en avances, el comienzo del trabajo de campo; ven la imposición de un esquema teórico como una limitante, lo que nos llevaría a tener un contacto reducido con la perspectiva de la investigación, se considera importante formular teorías en combinación con la recolección de datos, no antes. 


\section{REFERENCIAS CONSULTADAS}

1. Arias, F. (2012). El proyecto de Investigación. Editorial Episteme. Venezuela.

2. Azuero Azuero, Á. (2019). Significatividad del marco metodológico en el desarrollo de proyectos de investigación. Revista Arbitrada Interdisciplinaria Koinonía, 4(8), 110-127. doi:http://dx.doi.org/10.35381/r.k.v4i8.274

3. Báez J .(2009). Investigación Cualitativa. Editorial ESIC .Libros Profesionales de Empresa

4. Báez, J. y Pérez, de T. (2009). Investigación Cualitativa. Segunda Edición. Editorial ESIC. Pozuelo de Alarcon. Madrid, España.

5. Domínguez (2000) Investigación cualitativa y psicología social crítica. Contra la lógica binaria y la ilusión de la pureza. Investigación cualitativa en Salud.

6. Guba (1990) El Paradigma Dialógico de Investigación. Ediciones Mc Graw Hill. México.

7. Hernández, Fernández y Baptista (2010) Metodología de la Investigación. Ediciones Mc Graw Hill. México.

8. Taylor y Bogdan (1992) Introducción a los métodos cualitativos de Investigación. Buenos Aires. Paidos.

9. Universidad Pedagógica Experimental Libertador (2006) Manual de Trabajo de Grado de Especialización y Maestría y Tesis Doctorales Fondo Editorial de la Universidad Pedagógica Experimental Libertador.

10. Vera; L (2015) La Investigación Cualitativa. Universidad Interamericana de Puerto Rico. Recinto de Ponce. 